\title{
Visualization of dynamic stress conditions in elastic solids utilizing high frequency stroboscopic LED arrays
}

Shi, Jian, Ohrdes, Hendrik, Weinstein, Michael, Twiefel, Jens

Jian Shi, Hendrik Ohrdes, Michael Weinstein, Jens Twiefel, "Visualization of dynamic stress conditions in elastic solids utilizing high frequency stroboscopic LED arrays," Proc. SPIE 11105, Novel Optical Systems, Methods, and Applications XXII, 1110503 (9 September 2019); doi: $10.1117 / 12.2529955$

SPIE Event: SPIE Optical Engineering + Applications, 2019, San Diego, California, United States 


\title{
Visualization of dynamic stress conditions in elastic solids utilizing high frequency stroboscopic LED arrays
}

\author{
Jian Shi, Hendrik Ohrdes, Michael Weinstein, Tim Wielert, Jens Twiefel \\ Leibniz Hannover University \\ Institute of dynamics and vibration research \\ Appelstr.11, 30167 Hannover, Germany
}

\begin{abstract}
Ultrasonic mechanical vibrations in solids are widely used in non-destructive testing, and high-power applications such as ultrasonic welding or soldering. The visualization of ultrasonic wave propagation in transparent solids is helpful for understanding the ultrasonic behaviours. The classical method of photoelasticity allows the visualization of the static stress distribution in birefringent materials. Utilizing recent high-power LEDs in the photoelasticity allows to capture dynamic stresses by high frequency stroboscopic light. High frequency stationary and transient oscillation processes in elastic solids can be visualized with this method. The designed LED array in this paper has a dimension of $210 \mathrm{~mm} \times 300 \mathrm{~mm}$, and every LED has distance of $38 \mathrm{~mm}$ to each other, and the light intensity has a homogeneity value. The temporal and spatial resolution of stress-optic systems depends mainly on the dynamic properties of the lighting technology used. The high speed synchronization of the stroboscopic light sources results in a high temporal resolution of the photoelasticity analyses. This enables the photoelastic investigation of highly dynamic load conditions, such as longitudinal waves and transverse waves.
\end{abstract}

Keywords: stroboscopic LED array, Ultrasound, IGBT, dynamic photoelasticity

\section{INTRODUCTION}

Ultrasonic techniques, such as ultrasonic welding, ultrasonic motors, and ultrasonic friction reduction, are widely investigated in research and applied in industry. ${ }^{1,2}$ The highly dynamic vibrations can be visualized through dynamic photoelasticity. Four dominant recording methods for dynamic photoelasticity are high speed framing camera, the Cranz-Schardin system, Q-switch ruby laser, and stroboscopic light system. ${ }^{3}$ Among these four methods, stroboscopic light system has the advantage of affordable price for high quality results. Becker initially investigated impact problems with a strobe light, which permits the recording of a dynamic event by a photoelastic camera. When the illumination is aligned in phase with the dynamic stress wave propagation, the stress distribution appears frozen. ${ }^{4}$ A stroboscopic light system is the key technology to synchronize the light pulse to the frequency of vibration. Light emitting diodes (LED), as light source, offer obvious advantages over traditional light sources, such as spark discharge stroboscope or commercial gas discharge stroboscope tube stroboscope, in high efficiency, optical output power, modulation dynamics of short pulse and frequency, thermal management, and price. ${ }^{5-7}$ A single LED may not be appropriate for the whole field of visualization. Instead LED arrays are used to provide large homogeneous light fields. This depends on the topology of the circuit to achieve a sufficient optical output power. Meanwhile, the current through the LED array should be switched fast enough and ensure stable control for each light pulse signal.

For studying of dynamic stress in transparent solid, this paper proposes an stroboscopic light system, which is based on IGBT driver control circuit to modulate frequency and pulse duration of the LED array. This paper first introduces the principle of photoelasticity, then the design of the LED array, and stroboscopic driver control circuit. The modulation of the pulses of the LED characteristics is achieved by measuring the current through LED. Finally, the stress gradient distributions with the designed stroboscopic light system are shown.

Further information:

E-mail: jian.shi@ids.uni-hannover.de

Novel Optical Systems, Methods, and Applications XXII, edited by Cornelius F. Hahlweg, Joseph R. Mulley, Proc. of SPIE Vol. 11105, 1110503 - (c) 2019 SPIE CCC code: $0277-786 \mathrm{X} / 19 / \$ 21 \cdot$ doi: $10.1117 / 12.2529955$ 


\section{EXPERIMENTAL METHOD AND DESIGN}

In this section, a stroboscopic light system for dynamic photoelasticity is introduced. Dynamic photoelasticity, as measurement principle, will be first presented. The LED array is designed to provide a homogeneous light together with a polariscope, which can provide a whole field stress distribution. A custom designed LED driver circuit was built to modulate the frequency of light pulse.

\subsection{Principle of photoelasticity}

Photoelasticity is an effective method to study inner stress or strain inside transparent birefringent materials. When a birefringent material is under stress, it shows double refraction characteristics. This effect resolves incident polarized light into two components, respectively along a fast and a slow axis. Because of the velocity difference, there is a relative phase shift. The two components interface each other after passing through the birefringent material. The principle stress and the refractive index can be expressed in the stress-optic law: ${ }^{8}$

$$
\sigma_{1}-\sigma_{2}=\frac{N f_{\sigma}}{h}
$$

where

$$
f_{\sigma}=\frac{\lambda}{C}
$$

$\sigma_{1}-\sigma_{2}$ is the principal stress difference. $\mathrm{N}$ is the fringe order, $\mathrm{C}$ is the stress-optic coefficient $\mathrm{h}$ is the thickness of the material. $f_{\sigma}$ is the dynamic material stress fringe value. For dynamic situations, $f_{\sigma}$ value is generally $10 \%-30 \%$ higher than the static value of the material. ${ }^{9}$

Dynamic photoelasticity is often used for transient stress analysis, which requires correct synchronization of dynamic oscillations with stroboscopic light. For dynamic photoelastic studying, the exposure time depends on the speed of the stress waves in solids. To capture a longitudinal stress wave with speed of $2000 \mathrm{~m} / \mathrm{s}$, the exposure time should less than one microsecond. Some dynamic cameras can achieve this fast framing rate. ${ }^{8}$ In this paper, the stroboscopic imaging technique is used to visualize stress distribution induced by ultrasound wave in transparent polycarbonate. The light source for stroboscopic technique will be discussed in the following sections.

\subsection{LED module}

The LED module is specifically designed for use for photoelastic visualisation. The selected size of the illuminated area is chosen to be close to an DIN A4 format $(210 \mathrm{~mm} \times 300 \mathrm{~mm})$. The main target of its design is a homogeneous light distribution to minimize a possible source of misinterpretation due to non-uniform light distribution in the resulting photoelastic images. To achieve the most homogeneous light distribution, several parameters in the design have to be considered. Those include the opening angle of the LEDs, the heat dissipation, the diffuser used and its distance to the LEDs. Even if the LEDs are mounted on an actively cooled heat sink, their number is limited by their heat dissipation. The maximum number of LEDs results from the quotient of the maximum heat to be dissipated by the heat sink and the amount of heat generated per LED. With the condition that M-by-N equally spaced LED shall be used, the maximum total number of LEDs is calculated, according to

$$
n=\frac{\theta_{\mathrm{i}}-\theta_{\mathrm{u}}}{R_{\mathrm{thK}} \cdot U_{\max } I_{\max }}-\frac{R_{\mathrm{thG} i}+R_{\mathrm{thM} i}}{R_{\mathrm{thK}}},
$$

where $\theta_{\mathrm{i}}=150^{\circ} \mathrm{C}$ is the maximal junction temperature, $\theta_{\mathrm{u}}=45^{\circ} \mathrm{C}$ is the ambient temperature, $R_{\mathrm{thK}}=0.3 \mathrm{~K} / \mathrm{W}$ is the heat resistance of the heat sink, $U_{\max }=3.5 \mathrm{~V}$ is the maximal driving voltage, $I_{\max }=1.4 \mathrm{~A}$ is the maximal driving current, $R_{\mathrm{thG} i}+R_{\mathrm{thM} i}=5 \mathrm{~K} / \mathrm{W}$ is the sum of all 40 LEDs thermal inner LED and surface connection resistances. This leads to maximum of 41 LEDs that can be driven safely. The matrix chosen is a 8-by-5 LED matrix, which results in a distance of $38 \mathrm{~mm}$ between the LEDs. The next step is the evaluation of the optimal distance between the LEDs and the diffuser. To evaluate this, the light distribution is examined. In the model the light distribution of all LEDs is calculated and superimposed. Additionally, mirrors at the edges of the 
module are simulated to achieve a more homogeneous distribution at the edges of the illuminated plane. The distribution is calculated by ${ }^{10,11}$

$$
\begin{aligned}
E_{\mathrm{rel}}(x, y, z) & =z^{m} \sum_{i=1}^{N} \sum_{j=1}^{M}\left\{\left[x-(N+1-2 i) \frac{d}{2}\right]^{2}\right. \\
& \left.+\left[y-(M+1-2 j) \frac{d}{2}\right]^{2}+z^{2}\right\}^{-\frac{m+2}{2}}
\end{aligned}
$$

where $E_{\text {rel }}$ is the relative illumination, $x, y, z$, are coordinates, while $d$ is the distance, $M$-by- $N$ is the LED matrix size, and

$$
m=\frac{-\ln 2}{\ln \left(\cos \theta_{1 / 2}\right)}
$$

is a value that considers the opening angle $\theta_{1 / 2}$ of the LEDs. The results are then used to calculate the homogeneity that is defined as

$$
h=\frac{E_{\min }}{E_{\max }} .
$$

A variation of the distance $d$ provides the optimal distance for a most homogeneous light distribution. The design for the LED module is shown in Fig. 1. LEDs are mounted on the the cooling block, and on the top with a distance of $\mathrm{z}$ is diffusion screen. The mirror is used to reflect and restrict the light in the light module, even the boundary of the LED module has a homogeneous light distribution.

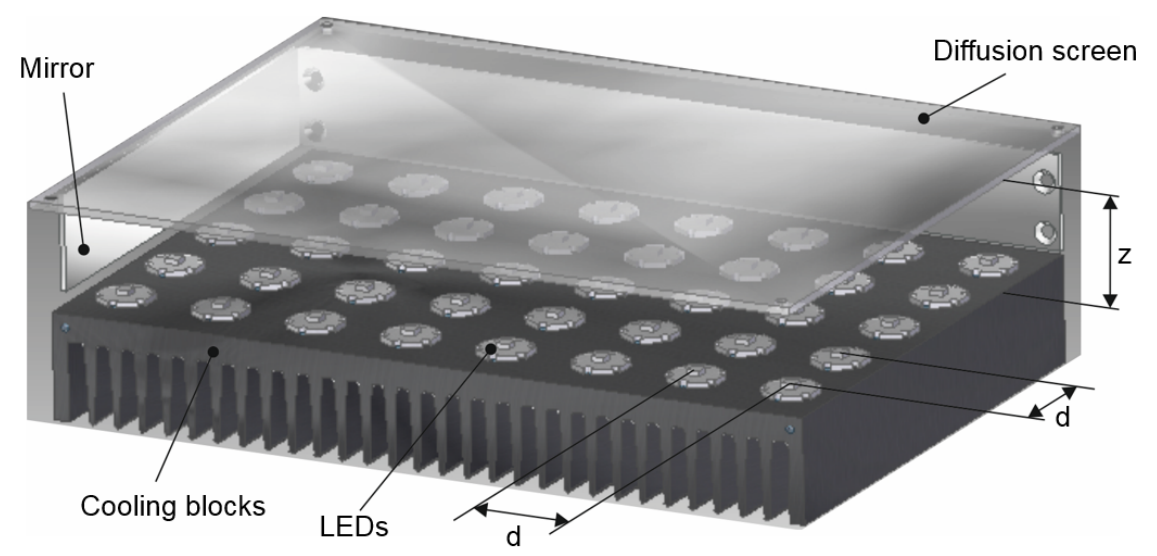

Figure 1: Concept design for the LED module

\subsection{Light distribution of the LED module}

The results of the simulation utilizing the model described in section 2.2 as well as a camera based experimental validation are shown in Fig. 2. This figure depicts the relation between the distance between the LEDs and the diffuser and the resulting relative homogeneity. It can be observed that there is an optimal distance that leads to the most homogeneous distribution, which is located around $d=38 \mathrm{~mm}$ for both the model and the experiment. In the simulation results, the diffusion screen effect on the light homogeneity was not considered. This explains the curve didn't fit to the experiment results. Due to results of experiment, a distance of $38 \mathrm{~mm}$ is used for the final setup. Fig. 3 shows the final light distribution as a picture and evaluated picture of the final setup with a distance of $38 \mathrm{~mm}$. The homogeneity of the distribution is calculated to be above $97 \%$, which is a sufficient value, so the influence of the light distribution has a minimal effect on the photoelastic experiments. 


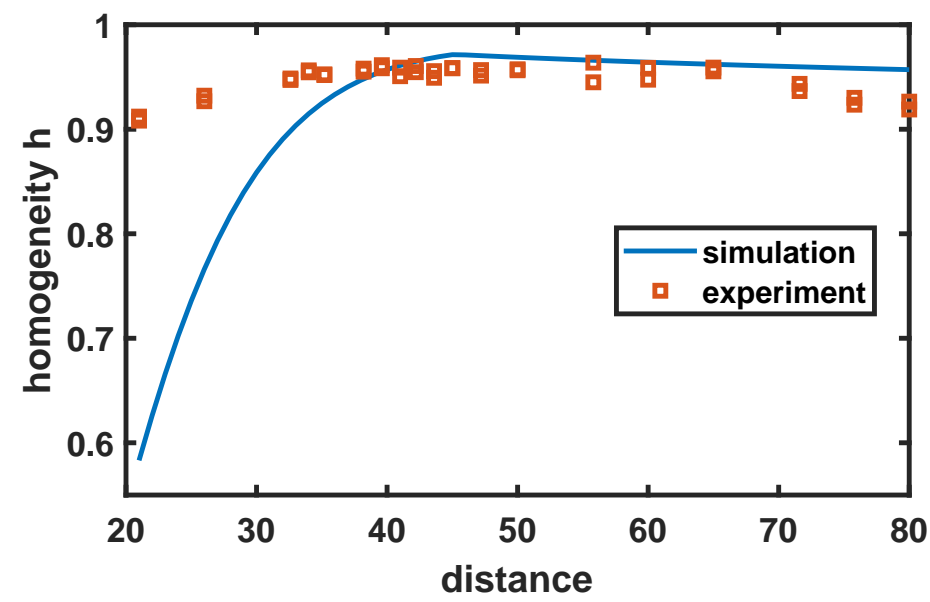

Figure 2: Relation between distance and homogeneity of the illuminated surface
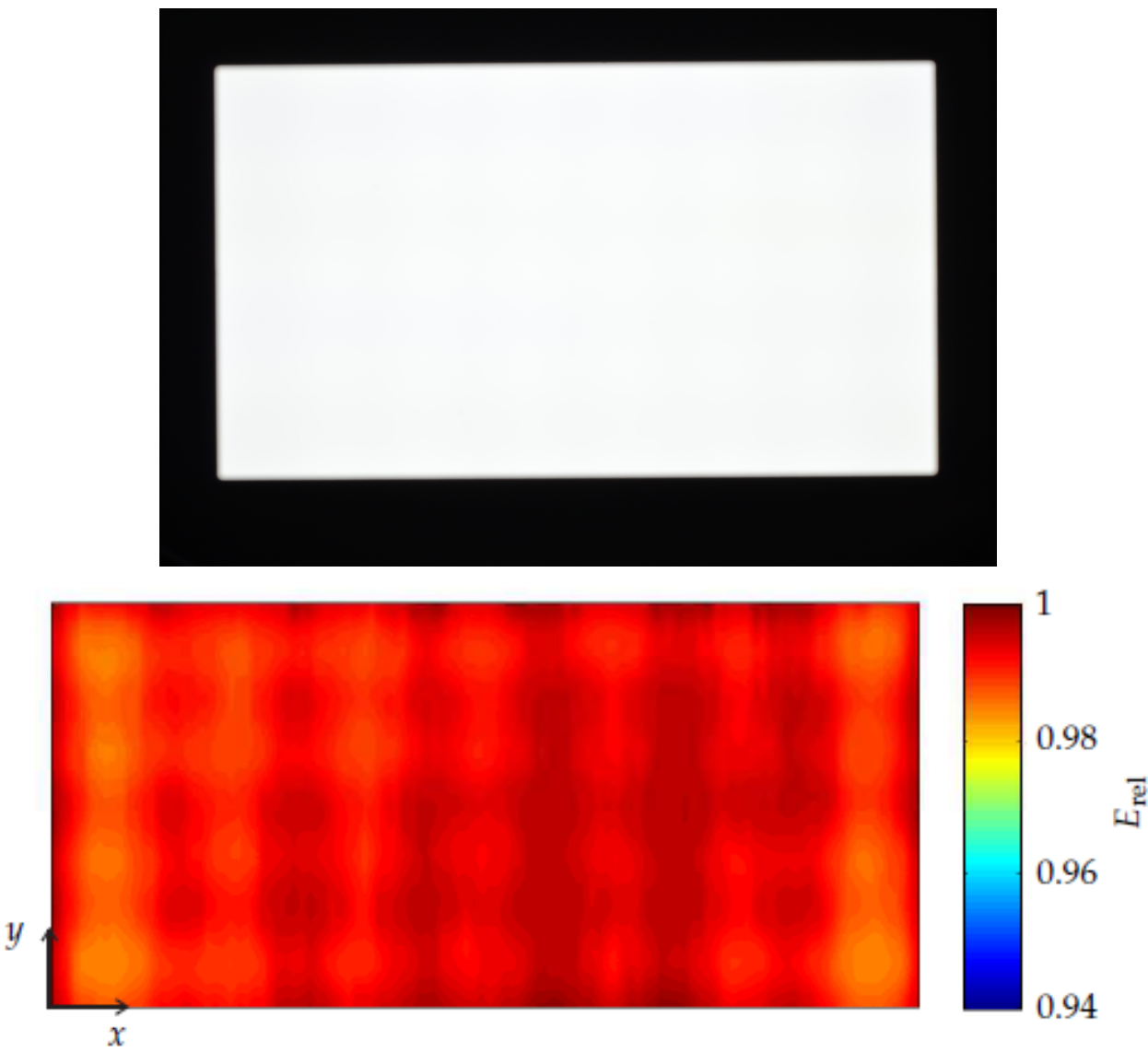

Figure 3: Picture of the light source and evaluation of the light distribution

\subsection{Design of IGBT control circuit}

For driving high frequency stroboscopic LEDs, transistors or Metal Oxide Field Effect Transistor (MOSFET) are triggered by pulses from a function generator at a certain frequency and pulse width. ${ }^{12,13} \mathrm{~A}$ MOSFET is a field effect transistor where the gate is insulated with a silicon oxide, which isolates the gate control signal from the higher power current flow through the device and operates the high current with small current and voltage. 
Insulated Gate bipolar Transistor (IGBT) is essentially a MOSFET controlling bipolar junction power transistor with both transistors integrated on a single silicon. Due to their ability to switch high currents and voltages, IGBTs are used in the introduced LED driving circuit. Fig.4a shows the topography of the designed circuit, and the variation of the light pulse width and switching frequency of light pulse is controlled externally by function generator. It consists of four functional modules. The voltage regulator module is in charge of providing the stable voltages of $5 \mathrm{~V}$ and $15 \mathrm{~V}$ for the supply of other circuit components. The Optocoupler (HCPL2630) isolates the signal of function generator from the driving signal. The control signal passes further through the IGBT driver (IXDI609) to the gates of the IGBT switches. IXDI609 has low propagation delay of 60ns, a fast rise time of 35ns and a short fall time of 25ns. G1 and G2 are IGBT (STGBL6NC60DI) from ST microelectronics, which have the ability to endure $6 \mathrm{~A}$ and a maximum voltage of $600 \mathrm{~V}$. The components were soldered onto a printed circuit board (PCB) as shown in Fig. 4b. The driving circuit is supplied by external high voltage source. The developed LED driver circuit allows to vary the switching frequency and the power of the LED array independently $(\mathrm{S} 2)$. For the present investigation only the varies of frequency is examined. The is the reason why G2 is set to high level permanently. The intensity is varied by the duty cycle of the input signal S1.

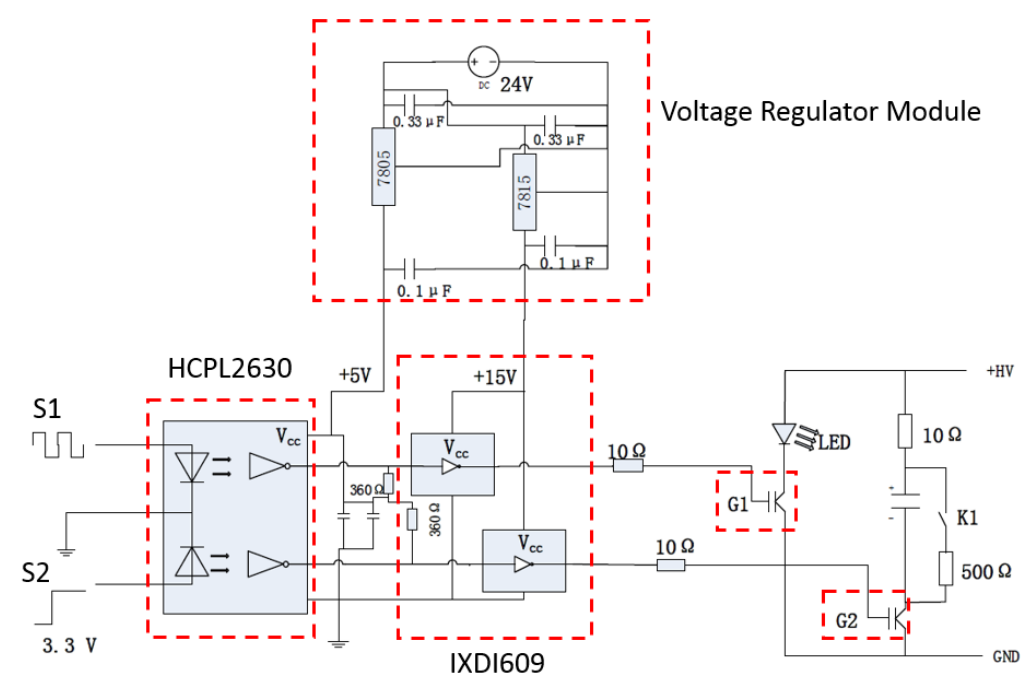

(a) Design of the stroboscopic LED control circuit

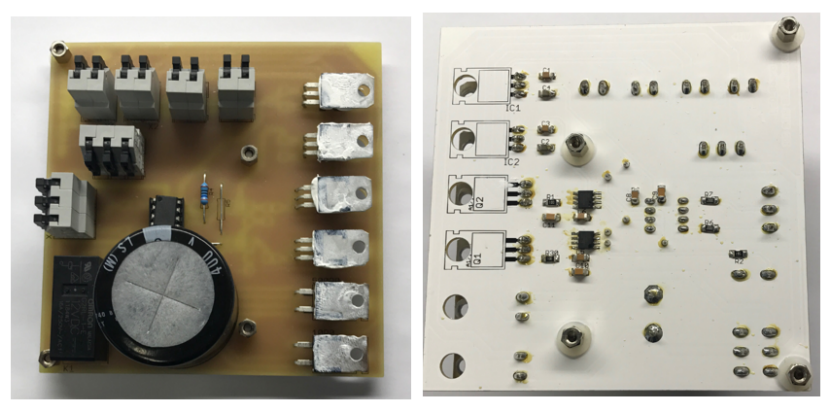

(b) PCB board for stroboscopic LED driver circuit

Figure 4: Integrated PCB control circuit for stroboscopic LED array

\subsection{Stroboscopic imaging system}

The experimental setup is illustrated as Fig.5. The stroboscopic circuit driver is powered by a DC high voltage supplier and a $24 \mathrm{~V} \mathrm{DC}$ source. The high voltage supplier is in charge of the LED and the $24 \mathrm{~V}$ DC source is powering for the voltage regulation of driver circuit. The function generator is used to control the frequency and 
pulse width. The stroboscopic module contains the stroboscopic LED control circuit and drives the LED array. A polycarbonate excited by ultrasound transducer is placed between the polariscope. A digital camera is placed on the other side of polariscope. In case of transient visualization, a high speed camera can be used to capture the transient wave propagation in solids.

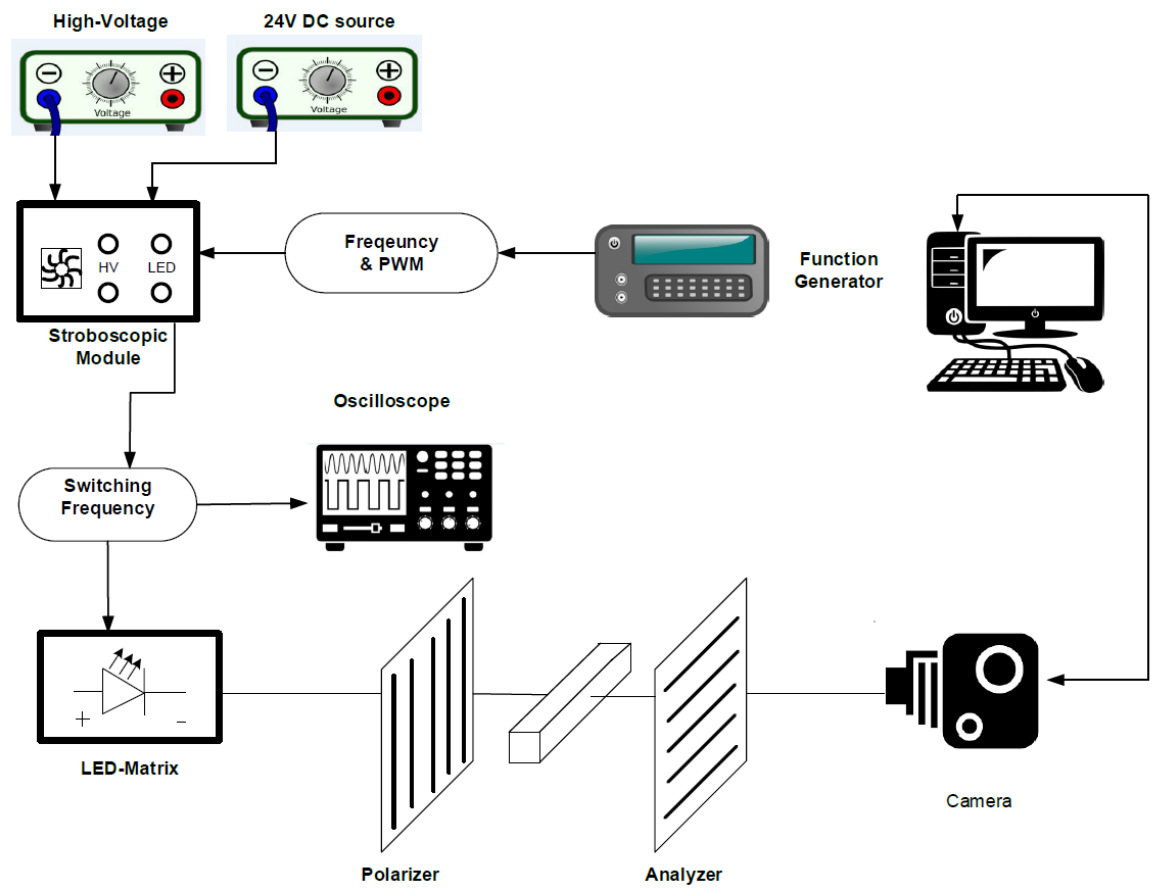

Figure 5: Illustrated experiment setup for stroboscopic imaging system

\section{RESULTS AND DISCUSSION}

In this section, the light distribution of the LED array and the current through LED arrays will be presented. The dynamic stress distribution induced by longitudinal ultrasound inside the transparent polycarbonate during different phase will be shown.

\subsection{Stroboscopic characteristics of the light source}

In theory, the brightness of LED increases linearly with the current. However, the continuous operation of LED leads to thermal effect, therefore the brightness-current characteristics of LED is non-linear under continues current state conditions. Gunarathne showed that, LED relative brightness and forward current are in linear relation, and more efficient in pulsed operation mode than in continuous mode. ${ }^{7}$ The pulsed operation mode allows LEDs to cool down between adjacent pulses. Considering in a cycle pulse operation, the average of pulsed mode LED are relative low, however the peak power can reach higher values than the limited current in continuous operation, ${ }^{13}$ as shown in Eq. 7. $P_{\text {avg }}$ is the average power of LED, and $P_{\text {peak }}$ is the peak power of pulse, and $\mathrm{W}$ is the pulse duty cycle.

$$
P_{\text {avg }}=P_{\text {peak }} \cdot W
$$

Through a current probe, the current passing thorough the LED array was measured, and the current status reflects the pulse effect of the LED array. Fig. 6 shows the current through LED array controlled by driving circuit. $20 \mathrm{kHz}$ and $70 \mathrm{kHz}$ of the repeating light pulse are selected to show the ability of the driving circuit. The rise time of the pulse is $1 \mu \mathrm{s}$, and the fall time of the pulse is $50 \mathrm{~ns}$. This presents fast switching on and off ability. Pulses duration is $t=1 / f \cdot W, \mathrm{f}$ is the frequency set by function generator, $\mathrm{W}$ is the pulse width. For the case of $20 \mathrm{kHz}$ and $70 \mathrm{kHz}$, the time between two adjacent pulse are $50 \mu s$ and $14 \mu s$. The pulse frequency can be precisely controlled with a sharp rising and falling edge. On the right side of the figure, the various width of pulse are also demonstrated from $2 \%$ to $20 \%$, which proved the pulse width can be precisely controlled. 


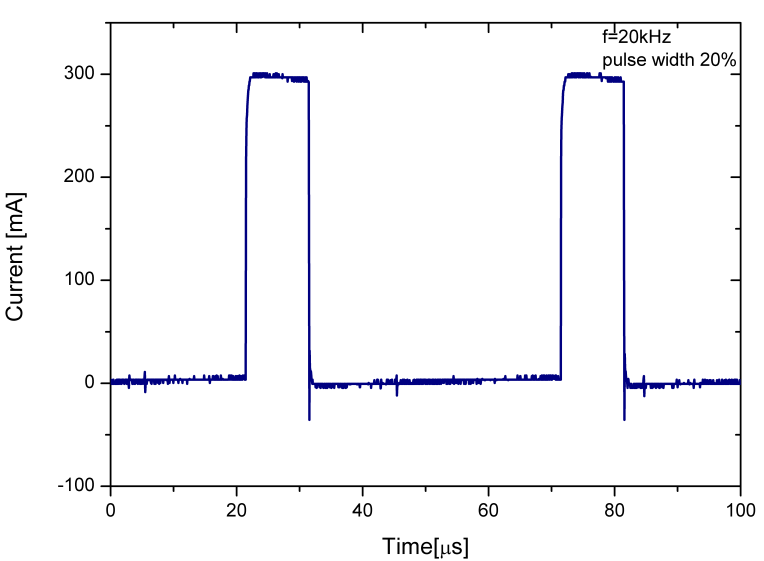

(a)

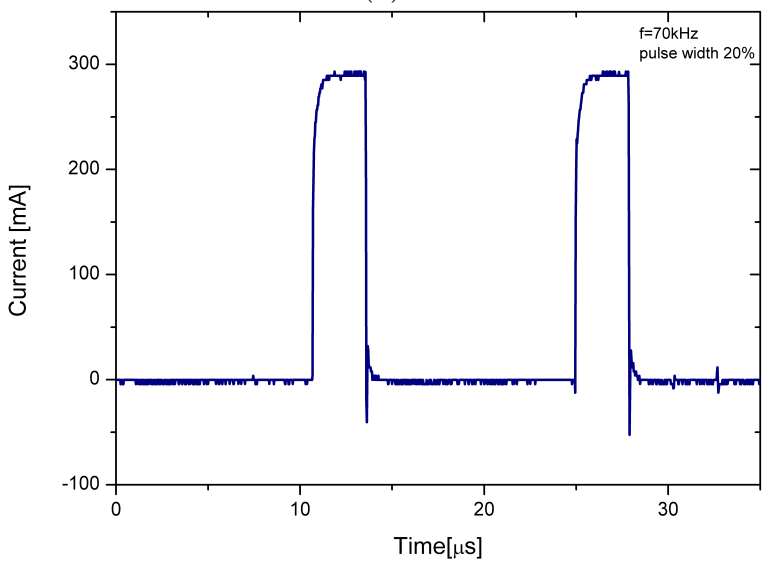

(c)

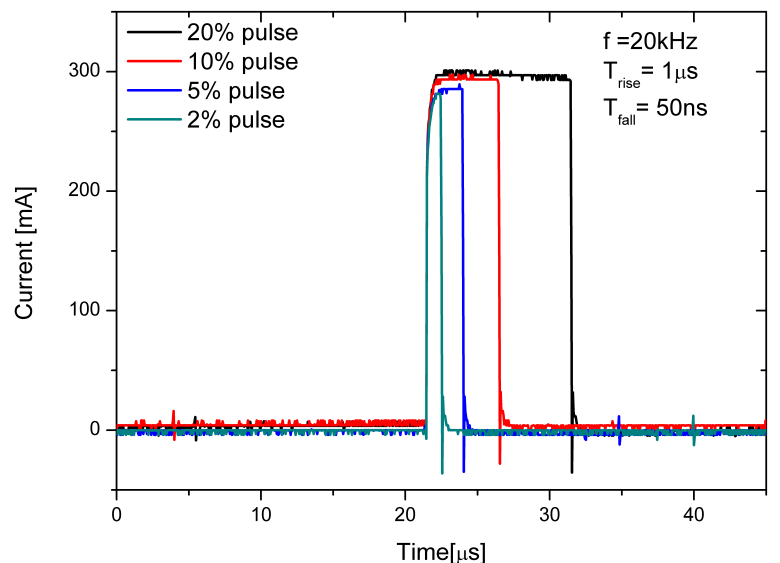

(b)

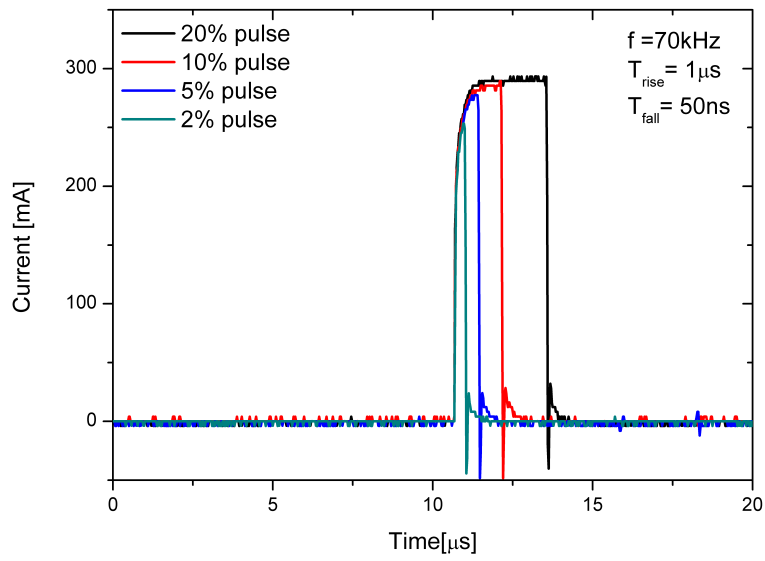

(d)

Figure 6: Modulation of light pulse width and frequency. (a) 20kHz with $20 \%$ of the whole cycle (b)Modulation of $20 \mathrm{kHz}$ pulse widths (c) $70 \mathrm{kHz}$ with $20 \%$ of the whole cycle (d)Modulation of $70 \mathrm{kHz}$ pulse widths

\subsection{Dynamic stress distribution in solid}

To couple ultrasonic waves in solid, a ultrasonic transducer is used and connected with the solid as shown in Fig. 7. The ultrasonic transducer consists of four PZT ceramics(PIC-181) and aluminium parts. The excited frequency of the ultrasonic transducer is provided by frequency function generator. Ultrasonic longitudinal waves are expected to propagate in axis direction. Another frequency generator is used for driving the LED. When the light is synchronized to the vibration frequency of the ultrasonic transducer, stress distribution induced by ultrasonic wave appears. By adjusting the phase between the ultrasonic driving signal and the pulsed light signal,

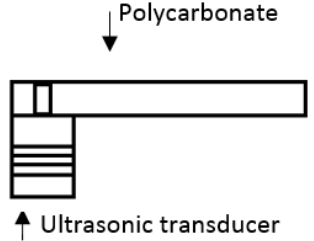

(a)

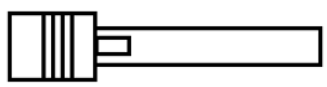

(b)

Figure 7: Experimental setup for transparent solid and ultrasonic transducer

the stress distribution in transparent solid can be visualized. The scheme for synchronizing of pulsed light and 
the vibration frequency of ultrasound in solids is shown in Fig. 8a. The red part indicates the light pulse, and the blue curve indicates the ultrasonic signal excited in ultrasonic transducer. When the light pulses and the driving signal of the transducer are in phase, the fringe pattern appears stationary. The grey part indicates the phase shift between pulsed light and the ultrasonic signal generated in the ultrasonic transducer. By adjusting the phase from 0 to $\pi / 2$, the various status of ultrasonic wave can be recorded with the digital camera. This method is limited to reproduced vibration. And Fig. 8b provides the rapid identification of stress gradients zones with different colours induced by ultrasonic waves in solids. The relation between the photoelastic colour and the stress inside the solid can found in Michel-Lvy interference colour chart.

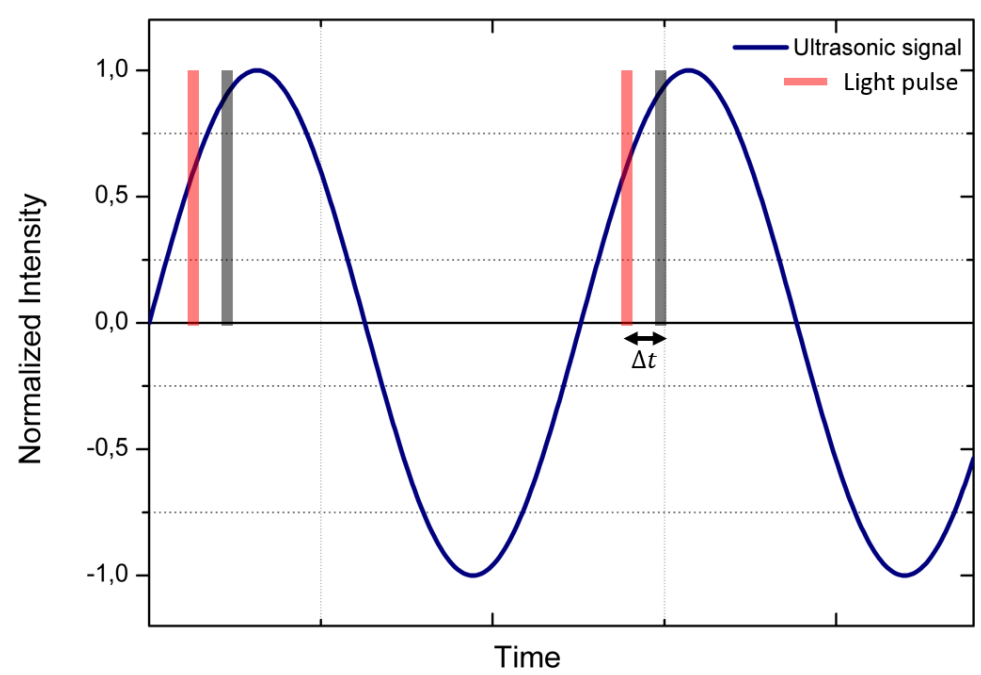

(a) Scheme for synchronizing of light signal to the vibration frequency of ultrasound in solid

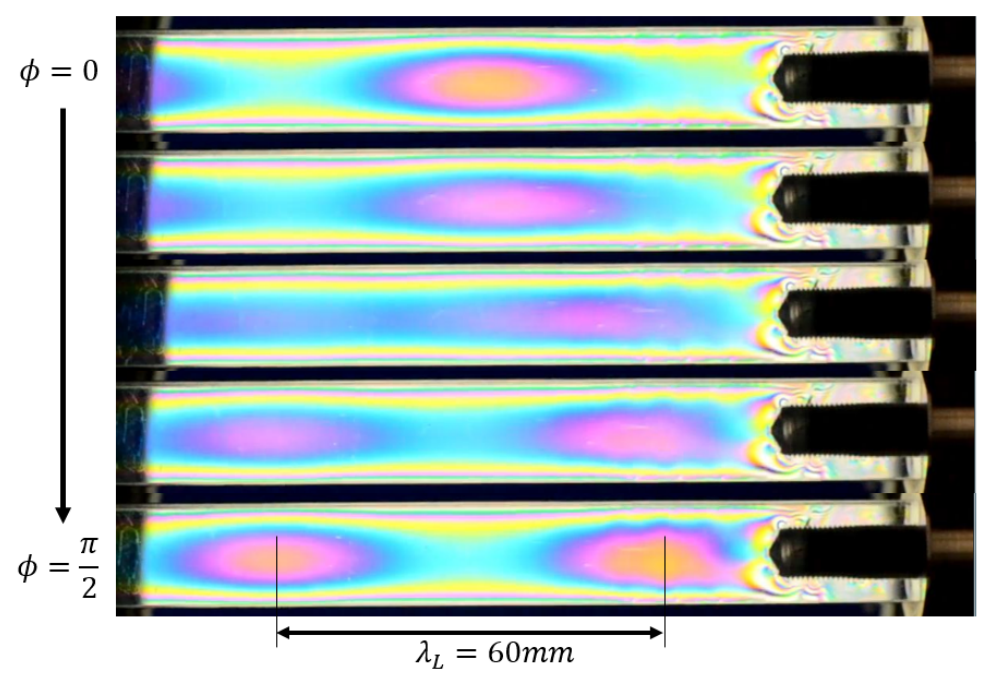

(b) Stress distribution induced by ultrasound wave in polycarbonate at various phase shift

Figure 8: Visualization of ultrasound wave with frequency of $23.6 \mathrm{kHz}$ in polycarbonate. 


$$
\lambda=\frac{1}{f} \sqrt{\frac{E}{\rho}}
$$

For the birefringent material with density of $\rho$ and Young's modulus E, driven by a frequency $f$, the longitudinal wavelength of the stress wave can be calculated according to Eq. 8. From the data sheet of the polycarbonate, the density of polycarbonate is $1.2 \times 10^{3} \mathrm{~kg} / \mathrm{m}^{2}$, Young's modulus is $2400 \mathrm{MPa}$. The excited frequency for ultrasonic transducer is $23.6 \mathrm{kHz}$. The measured value is about $60 \mathrm{~mm}$, and the theoretical calculated wavelength $59.9 \mathrm{~mm}$ of longitudinal wave. The pattern shown in Fig. $8 \mathrm{~b}$ can be regard as the longitudinal wave inside the polycarbonate. However, this formula should be carefully used, it is suits for long and thin beam. For other shapes of solids, the wavelength may vary. Not only the longitudinal wave can be visualized, the transverse wave with frequency of $22.8 \mathrm{kHz}$ in polycarbonate is shown in Fig. 9. This method can be used for rapid identifying stress gradients. There are many mature stress image techniques for photoelasticity. In this paper, the stress distribution will not be further discussed.

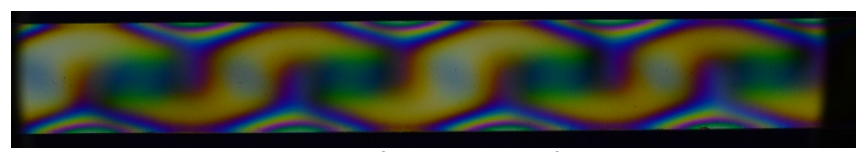

Figure 9: Transverse wave with frequency of $22.8 \mathrm{kHz}$ in polycarbonate.

\section{CONCLUSION}

The stroboscopic light system for dynamic stress visualization is introduced in this paper. The frequency and pulse duration of light pulse is demonstrated to be precisely controlled. The rise time of the pulse is about $1 \mu s$, and the fall time is about $50 \mathrm{~ns}$. This shows fast switching on and off ability to operate the light pulse. When the frequency of light pulse is synchronized with the frequency of ultrasonic stress wave in transparent solid, the wave pattern appears stationary. The stress induced by ultrasonic wave in polycarbonate is demonstrated by stroboscopic LED. The stroboscopic light system can be used to visualize the stress distribution induced by ultrasound propagation in birefringent solids. In the following work, the stress distribution of ultrasound will be analysed.

\section{ACKNOWLEDGMENTS}

This project is within the Tailored Light Programme funded by Lower Saxony Ministry of Education.

\section{REFERENCES}

[1] N.Cheeke, J., [Fundamentals and Applications of Ultrasonic Waves], Taylor \& Francis Group, LLC, second edi ed. (2012).

[2] Kuttruff, H., [Ultrasonics: fundamentals and applications], ELSEVIER SCIENCE PUBLISHERS LTD (1991).

[3] Clark, J. a. and Durelli, a. J., "An introduction to dynamic photoelasticity," Experimental Mechanics 23(1), $42-48$ (1983).

[4] Becker, H., "Simplified Equipment for Photoelastic Studies of Propagating Stress Waves," Experimental Mechanics 1(12), 214-216 (1961).

[5] Andrews, D. R. and Wallis, L. J., "Light emitting diode as a short-duration stroboscope-application to visualisation of ultrasound," Journal of Physics E: Scientific Instruments 10(1), 95 (1977).

[6] Hall, K. G., "Observing ultrasonic wave propagation by stroboscopic visualization methods," Ultrasonics 20(4), 159-167 (1982).

[7] Gunarathne, G. P. P. and Szilard, J., "A new stroboscope for schlieren and photoelastic visualization of ultrasound," Ultrasonics 21(4), 188-190 (1983). 
[8] Sharpe, ed., [Springer Handbook of Experimental Solid Mechanics], vol. XXXIII (2012).

[9] Dally, J. W., "An introduction to dynamic photoelasticity," Experimental Mechanics 20(12), 409-416 (1980).

[10] Moreno, I., Avendaño-Alejo, M., and Tzonchev, R. I., "Designing light-emitting diode arrays for uniform near-field irradiance," Applied optics 45(10), 2265-2272 (2006).

[11] Whang, A. J.-W., Chen, Y.-Y., and Teng, Y.-T., "Designing uniform illumination systems by surfacetailored lens and configurations of led arrays," Journal of display technology 5(3), 94-103 (2009).

[12] Paulin, T., Heikkinen, V., Kassamakov, I., and Hæggström, E., "Led driver for stroboscopic interferometry," in [Optical Micro-and Nanometrology IV], 8430, 84301A, International Society for Optics and Photonics (2012).

[13] Winder, S., [Power supplies for LED driving], Newnes (2016). 